\title{
Estudio comparativo entre la anamnesis y la ergonometría en la detección de limitaciones biomecánicas por sintomatologia musculoesquelética.
}

Jean Pino-Sánchez, Oscar Tapia-Claudio, Pamela Merino-Salazar y Yolis Campos-Villalta Universidad Internacional SEK, Quito, Ecuador.

Palabras clave: ergonometría; trastornos musculoesqueléticos; vigilancia de la salud; método biomecánico; ánǵulo de movilidad articular.

Resumen. El objetivo del presente estudio fue comparar las limitaciones biomecánicas encontradas, aplicando la anamnesis y la evaluación goniométrica, en trabajadores con síntomas musculoesqueléticos, y en otros asintomáticos, durante la evaluación médica periódica en una empresa ecuatoriana. Durante el 2018, se diagnosticaron y evaluaron 96 trabajadores administrativos y operativos-obreros, de una empresa manufacturera privada, mediante anamnesis y goniometría. Los resultados se compararon mediante la t de Student. En el 80\% de los segmentos evaluados se hallaron diferencias estadísticas significativas entre goniometría y anamnesis en ambos grupos (asintomáticos y sintomáticos) durante la evaluación médica periódica, con excepción en los movimientos de flexión e inclinación lateral de columna cervical, flexión de codo, desviación radial de muñeca y extensión de rodilla. Se concluye que la incorporación de la goniometría en el examen médico ocupacional podría contribuir a calificar la aptitud física del trabajador y a realizar un diaǵnóstico precoz de trastornos musculoesqueléticos. 


\title{
Comparative study between the anamnesis and ergonometry in the detection of biomechanical limitations due to musculoskeletal symptoms.
}

Invest Clin 2021; 62 (1): 52-62

Key words: ergonometry; musculoskeletal disorder; health surveillance; biomechanical method; joint mobility angle.

\begin{abstract}
The objective of the present study was to compare the biomechanical limitations found, applying the anamnesis and goniometric evaluation in workers with musculoskeletal symptoms, and in asymptomatic workers, during the periodic medical evaluation in an Ecuadorian company. During 2018, 96 administrative and operational workers of a private manufacturing company were diagnosed and evaluated through anamnesis and goniometry. The results were compared using the Student's t test. In $80 \%$ of the evaluated segments, significant statistical differences were found between goniometry and anamnesis in both groups (asymptomatic and symptomatic) during the periodic medical evaluation, with the exception of flexion and lateral inclination movements of the cervical spine, elbow flexion, radial wrist deviation and knee extension. It is concluded that the incorporation of goniometry in the occupational medical examination could contribute to qualify the physical fitness of the worker and to make an early diagnosis of musculoskeletal disorders.
\end{abstract}

Recibido: 10-07-2020 Aceptado: 18-12-2020

\section{INTRODUCCIÓN}

Dentro del campo de investigación más importante de la ergonomía, se encuentran las exigencias biomecánicas que demandan los puestos de trabajo en la población económicamente activa. Cuando las capacidades del trabajador son superadas por estas exigencias biomecánicas y los trabajadores no cuentan con una recuperación adecuada, pueden presentarse "Trastornos Musculoesqueléticos" (TME) (1).

Los TME son unas de las patologías más prevalentes en la población trabajadora a nivel mundial, y tienen un impacto económico significativo. Sin embargo, muchas de las enfermedades relacionadas con este tipo de trastornos cuyo origen es laboral, quedan encubiertas como enfermedades comunes (2).
Según la Encuesta Europea de Condiciones de Trabajo del 2005, el 24,7\% de la población trabajadora de los 27 países miembros de la Unión Europea refiere dolor de espalda (3). En una investigación realizada en Venezuela en el 2007 (4), se demostró una alta prevalencia de síntomas musculoesqueléticos, causada bien por las posturas forzadas, el manejo manual de cargas o por las operaciones con herramientas que realizan los trabajadores evaluados. Por otro lado, los datos de la Agencia Europea para la Seguridad y la Salud en el Trabajo (5) revelaron que el costo por TME en extremidades superiores en los Estados Unidos, supera anualmente los 2.100.000,00 dólares en indemnizaciones a los trabajadores mientras que los trastornos dorso lumbares equivalen a otros 11.000 millones de dólares por el mismo concepto. 
En Colombia, entre los años 20012004, el Ministerio de Protección Social reportó los TME como primera causa de enfermedad profesional (1). Por su parte, el Ecuador cuenta con normativa relacionada a la seguridad y salud en el trabajo, como el Decreto 2393 (6), el cual plantea la prevención, disminución o eliminación de los riesgos y el mejoramiento del medio ambiente de trabajo.

De igual forma, se cuenta con el Acuerdo Ministerial 1404 (7), donde el objetivo fundamental es mantener la salud integral del trabajador, que se traduce en un estado de bienestar físico, mental y social del mismo; y hace mención en el capítulo IV, artículo 11. 1. c "El análisis y clasificación de puestos de trabajo, para seleccionar al personal, en base a la valoración de los requerimientos psicofisiológicos de las tareas a desempeñar, y en relación con los riesgós de accidentes del trabajo y enfermedades profesionales"; en el artículo 11.2. a "Apertura de la ficha médica ocupacional al momento del ingreso de los trabajadores a la empresa, mediante el formulario que al efecto proporcionará el Instituto ecuatoriano de seguridad social (IESS)"; 11.2. b "Examen médico preventivo anual de seguimiento y vigilancia de la salud de todos los trabajadores".

No obstante, la escasa evidencia disponible sobre las patologías musculoesqueléticas en la población trabajadora del Ecuador, sugiere que los TME de origen laboral constituyen un problema de gran magnitud. Las estadísticas obtenidas de la Dirección Actuarial y de Investigación del Instituto Ecuatoriano de Seguridad Social (8) señalan que, a nivel nacional, el 3,83\% de la población afiliada padece de TME, lo cual provoca un 19,15\% de ausentismo laboral; ocasionando una reducción de ingresos y un elevado gasto para las empresas y para la economía nacional en el campo de la salud pública y privada.

Un estudio realizado por Oliveira y Rodríguez (9) en trabajadores administrativos del Ecuador, reportó que los TME se pre- sentan en el 6\% de la población, siendo el seǵmento corporal más afectado el tronco, y, las patologías más comunes en orden de frecuencia, las lumbalgias, radiculopatías y cervicalgias. Sin embargo, merecen especial atención los hallazǵos de un estudio transversal, basado en datos de la Primera Encuesta de Condiciones de Trabajo de Quito-Ecuador del 2016 (10), donde reportaron que el $56 \%$ de las mujeres y el $43,5 \%$ de los hombres refirieron dolor o molestias en la espalda.

La incorporación de técnicas de evaluación clínica que ayuden a detectar en forma precoz, limitaciones de los movimientos de algún segmento corporal, es un valor agregado que debemos considerar al momento de realizar el examen médico de ingreso y periódico anual de seguimiento y viǵilancia de la salud de los trabajadores, contemplado dentro de la normativa nacional.

En tal sentido, dentro de las técnicas que muchos médicos ocupacionales están incorporando en el examen físico, se encuentra la goniometría, que es una técnica práctica y económica, que está siendo aplicada en algunos países de Latinoamérica.

La goniometría se refiere a la medición de ángulos que generarán los huesos del cuerpo en las articulaciones, determinando tanto la posición de una articulación como su movilidad total. La práctica de movimientos articulares activos y pasivos durante la exploración física al trabajador, permite al examinador detectar movimientos anormales, medir y documentar el grado de movilidad articular, así como las posiciones fijas anormales de la articulación, a la vez que proporciona información sobre la presencia, ausencia o cambios en el deterioro de la articulación, diaǵnóstico, pronóstico y permite valorar progresos en la rehabilitación y modificar tratamiento, entre otros (11).

La goniometría es una técnica que permite obtener criterios para evaluar la capacidad biomecánica del trabajador y las exigencias físicas del puesto de trabajo; asimismo, permite evaluar la función motora periférica 
y musculoesquelética de un paciente con incapacidad física (12); y permite diagnosticar precozmente los problemas musculoesqueléticos. En tal sentido, en algunos países de Latinoamérica como Venezuela, dentro del marco técnico-normativo de la salud ocupacional, se establece la medición goniométrica como parte del examen médico de ingreso a todos los trabajadores (13). Sin embargo, en el Ecuador esta técnica es poco conocida y no existen investigaciones ni normativa legal que la contemple.

Aun cuando existen diferentes instrumentos que miden la movilidad articular y la longitud muscular, como los inclinómetros, electrogoniómetros, cámaras fotográficas y más recientemente, aplicaciones para goniómetros con teléfonos inteligentes, es el goniómetro universal el instrumento más usado para medir la movilidad articular en el marco clínico (11).

Por lo anterior, el presente estudio pretende comparar las limitaciones biomecánicas encontradas en los trabajadores de una empresa ecuatoriana en el año 2018, durante la evaluación médica periódica, al aplicar la goniometría, con la información recolectada durante la anamnesis de aquellos que refieren encontrarse asintomáticos y aquellos que refirieron presentar síntomas musculoesqueléticos.

\section{MATERIAL Y MÉTODOS}

Estudio de campo, transversal y comparativo, realizado a una población de 96 trabajadores de las áreas administrativa y operativa-obrera, de una empresa privada ecuatoriana dedicada al sector manufacturero, a quienes se les realizaron evaluaciones médicas periódicas durante el año 2018.

Se recogieron los antecedentes familiares, patológicos y ocupacionales de los últimos 5 años de cada paciente dentro de la historia médico ocupacional. Luego se realizó la exploración funcional del sistema musculoesquelético, presentando al médicoevaluador unas imágenes orientadoras como se muestra en la Fiǵ. 1 para la realización de la misma.

Se utilizaron tres tipos de goniómetros: universal, de pared y de mano, los cuales permiten evaluar la posición de las articulaciones en los tres planos espaciales (sagital, frontal y transversal).

Los valores de referencia considerados para la investigación son los descritos por Taboadela (12), pertenecientes a la Asociación para el Estudio de Osteosíntesis (AO) y los valores de $0-80^{\circ}$ de la Academia Americana de Cirujanos Ortopédicos (AAOS).

El examen goniométrico de la columna cervical se realizó con un goniómetro univer-

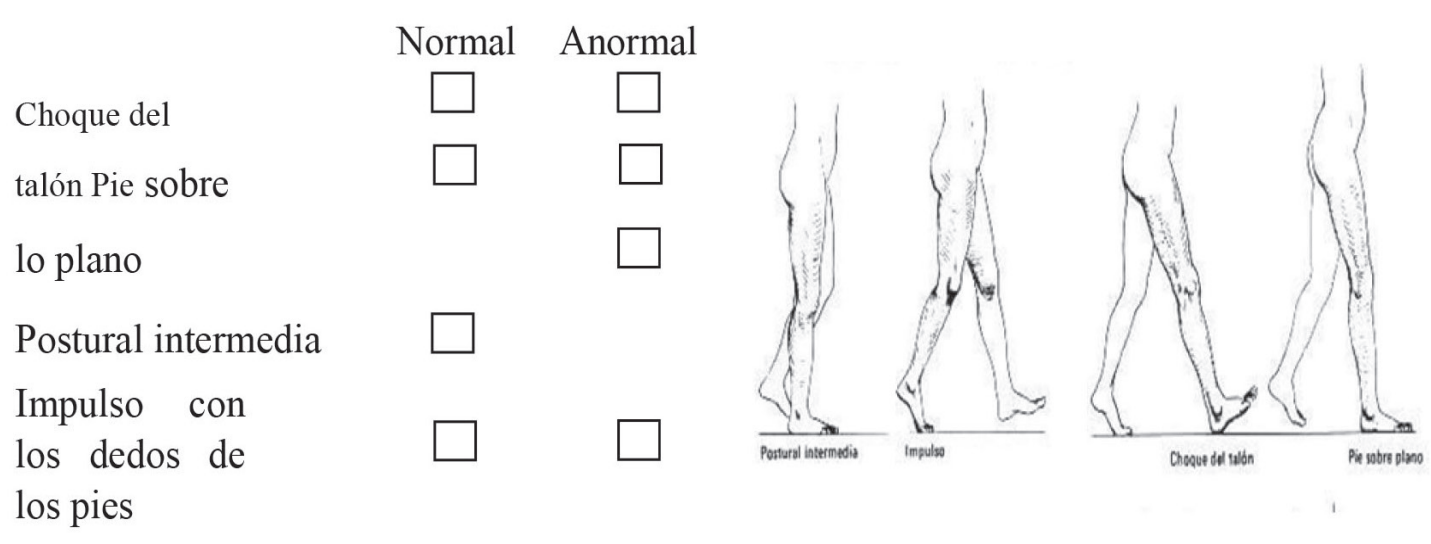

Fig. 1. Modelo de la Subsección Exploración Postural de la Marcha.

Vol. 62(1): 52 - 62, 2021 
sal y con los trabajadores en posición sentada. Los valores normales son de $0-35^{\circ} / 45^{\circ}$ para la flexión y extensión, $0-45^{\circ}(\mathrm{AO})$ para la inclinación lateral derecha e izquierda y $0-60^{\circ} / 80^{\circ}$ para la rotación derecha e izquierda.

Para el examen goniométrico de la columna lumbar, se usó el goniómetro de pared y con el trabajador de pie, espinas ilíacas anterosuperiores niveladas en la misma línea horizontal y perpendicular al piso. Los valores normales para la flexión son $0-80^{\circ}$ (AAOS), para la extensión $0-30^{\circ}(\mathrm{AO})$, para la inclinación lateral derecha e izquierda $0-30^{\circ} / 40^{\circ}(\mathrm{AO})$, y para la rotación derecha e izquierda $0-30^{\circ}(\mathrm{AO})$.

Por su lado, el examen goniométrico de miembros superiores (hombros y codos), se realizó con un goniómetro universal; mientras que, para evaluar la muñeca, se colocaron las manos sobre una superficie plana y se utilizó un goniómetro especial para la articulación condiloartrosis radiocarpiana, obteniendo los grados de desviación radial y cubital. En el caso del hombro, los valores normales son; para abducción $0-160^{\circ} / 180^{\circ}$ $(\mathrm{AO})$, aducción: $0-30^{\circ}(\mathrm{AO})$, flexión: 0-150 $(\mathrm{AO})$, extensión $0-40^{\circ}(\mathrm{AO})$, rotación externa: $0-70^{\circ}(\mathrm{AO})$ y rotación interna: $0-70^{\circ}$. Con respecto al codo, los valores normales son de $0-150^{\circ}(\mathrm{AO})$ para flexión y $0-10^{\circ}(\mathrm{AO})$ para extensión. Para la muñeca, los valores normales son: desviación radial $0-25^{\circ} / 30^{\circ}(\mathrm{AO})$ y desviación cubital $0-30 / 40^{\circ}(\mathrm{AO})$.

Finalmente, para realizar la goniometría de la rodilla en flexión, se colocó al trabajador en decúbito dorsal con el miembro inferior en posición $0^{\circ}$; para medir el movimiento en extensión, se colocó al trabajador en decúbito ventral con el miembro inferior en posición $0^{\circ}$ y el fémur estabilizado con una almohada colocada por debajo del mismo; siendo los valores normales de $0-150^{\circ}$ (AO) para la flexión, $0^{\circ}(\mathrm{AO})$ para extensión activa y $0-10^{\circ}(\mathrm{AO})$ para extensión pasiva.

Por último, se visualizó el estudio de imágenes ( $\mathrm{Rx}$ de tórax PA y lateral y $\mathrm{Rx}$ columna lumbo sacra AP y lateral).
Cada paciente firmó un consentimiento informado; y para ello escribió su número de cédula, estampó su rúbrica, y las huellas dactilares de los dedos pulgares.

Análisis estadístico. Se calcularon las medias y la desviación estándar de los rangos de movimiento articular tanto de los trabajadores sintomáticos como de los asintomáticos que acudieron a la evaluación médico ocupacional periódica. Se aplicó la t de Student para determinar las diferencias de medias entre los resultados de la goniometría y la sintomatología musculoesquelética reportada durante la anamnesis en la evaluación médica periódica. El paquete estadístico utilizado para el análisis de los datos fue el Statistical Package for the Social Sciences (SPSS), versión 22.0.

\section{RESULTADOS}

En la Tabla I se observan las características sociodemográficas de la población de estudio. El 68\% de la población trabajadora fueron del sexo masculino y el 32\% mujeres. Prevaleció el grupo de 25-34 años (36,9\%) con grado de instrucción de bachillerato $(56,9 \%)$.

La Tabla II detalla la localización de la sintomatología musculoesquelética que refirieron los trabajadores durante la anamnesis; 64 (67\%) pacientes negaron síntomas y 32 (33\%) pacientes relataron algún tipo de dolencias musculoesqueléticas. Se puede apreciar que el $27,7 \%$ de los hombres y el $45,2 \%$ de las mujeres reseñaron dolor musculoesquelético y en ambos sexos predominó el dolor lumbar, 9,2 \% en hombres y 19,4\% en mujeres, seguido de dolor cervical $6,2 \%$ en los hombres y $9,7 \%$ en mujeres.

En la Tabla III se muestra la evaluación médica periódica, donde se compara la anamnesis con los resultados de la medición goniométrica. De los 64 trabajadores (67\%) que manifiestaron encontrarse sin sintomatología musculoesquelética, al realizarles la evaluación goniométrica ninguno presentó 


\section{TABLA I}

DESCRIPGIÓN GENERAL DE LA POBLACIÓN DE TRABAJADORES DEL ÁREA ADMINISTRATIVA Y OPERATIVA

\begin{tabular}{lcccccc}
\hline & \multicolumn{2}{c}{ Hombres } & \multicolumn{2}{c}{ Mujeres } & \multicolumn{2}{c}{ Total } \\
\cline { 2 - 6 } & $\mathrm{n}=65$ & $\%$ & $\mathrm{n}=31$ & $\%$ & $\mathrm{~N}=96$ & $\%$ \\
\hline Edad & & & & & & \\
18-24 años & 27 & 41,5 & 13 & 41,9 & 40 & 41,7 \\
25-34 años & 24 & 36,9 & 7 & 22,6 & 31 & 32,3 \\
35-44 años & 4 & 6,2 & 4 & 12,9 & 8 & 8,3 \\
$45-54$ años & 5 & 7,7 & 2 & 6,5 & 7 & 7,3 \\
$\geq 55$ años & 5 & 7,7 & 5 & 16,1 & 10 & 10,4 \\
Grado Instrucción & & & & & & \\
Básica & 6 & 9,2 & 4 & 12,9 & 10 & 10,4 \\
Bachillerato & 37 & 56,9 & 16 & 51,6 & 53 & 55,2 \\
$3^{\circ}$ Nivel & 15 & 23,1 & 8 & 25,8 & 23 & 24,0 \\
$4^{\circ}$ Nivel & 7 & 10,8 & 3 & 9,7 & 10 & 10,4 \\
\hline
\end{tabular}

TABLA II

LOCALIZACIÓN DE LOS SÍNTOMAS MUSCULOESQUELÉTICOS SEGÚN LA ANAMNESIS.

\begin{tabular}{lcccccc}
\hline \multicolumn{1}{c}{$\begin{array}{c}\text { Síntomas } \\
\text { Musculoesqueléticos }\end{array}$} & Hombres & \multicolumn{3}{c}{ Mujeres } & \multicolumn{2}{c}{ Total } \\
\cline { 2 - 7 } \multicolumn{1}{c}{} & $\mathrm{n}=36$ & $\%$ & $\mathrm{n}=28$ & $\%$ & $\mathrm{~N}=64$ & $\%$ \\
\hline Cualquier segmento & 18 & 27,7 & 14 & 45,2 & 32 & 33,3 \\
Columna Cervical & 4 & 6,2 & 3 & 9,7 & 7 & 7,3 \\
Columna Lumbar & 6 & 9,2 & 6 & 19,4 & 12 & 12,5 \\
Hombro & 2 & 3,1 & 3 & 9,7 & 5 & 5,2 \\
Codo & 1 & 1,5 & 0 & 0,0 & 1 & 1,0 \\
Muñeca & 4 & 6,2 & 2 & 6,5 & 6 & 6,3 \\
Rodilla & 1 & 1,5 & 0 & 0,0 & 1 & 1,0 \\
\hline
\end{tabular}

TABLA III

COMPARACIÓN ENTRE ANAMNESIS Y GONIOMETRÍA EN TRABAJADORES CON Y SIN SÍNTOMAS MUSGULOESQUELÉTICOS.

\begin{tabular}{|c|c|c|c|c|c|c|c|c|}
\hline \multirow{3}{*}{ Patologías / Examen } & \multicolumn{4}{|c|}{ Anamnesis } & \multicolumn{4}{|c|}{ Goniometría } \\
\hline & \multicolumn{2}{|c|}{ Asintomáticos } & \multicolumn{2}{|c|}{ Sintomáticos } & \multicolumn{2}{|c|}{ Asintomáticos } & \multicolumn{2}{|c|}{ Sintomáticos } \\
\hline & $\mathrm{n}$ & $\%$ & $\mathrm{n}$ & $\%$ & $\mathrm{n}$ & $\%$ & $\mathrm{n}$ & $\%$ \\
\hline $\begin{array}{l}\text { Limitación de areos } \\
\text { de movilidad }\end{array}$ & & & 32 & 33 & & & 11 & 11,5 \\
\hline $\begin{array}{l}\text { Sin limitación de areos } \\
\text { de movilidad }\end{array}$ & 64 & 66,6 & & & 64 & 66,6 & 21 & 21,9 \\
\hline
\end{tabular}

Vol. 62(1): 52 - 62, 2021 
limitaciones en los arcos de movilidad, coincidiendo con lo reportado en la anamnesis. Sin embargo, de los 32 trabajadores que declararon en la anamnesis presentar sintomatología musculoesquelética, al efectuarles la medición goniométrica, solo 11 trabajadores presentaron limitaciones en los arcos de movilidad.

En la Tabla IV se observa el promedio y la desviación estándar de los resultados de medición de los areos de movilidad, aplicada al total de trabajadores. La media de los trabajadores que indicaron en la anamnesis encontrarse asintomáticos superó en todos los segmentos corporales, a la media de los trabajadores que refirieron encontrarse sintomáticos. En relación con la desviación estándar, en los trabajadores asintomáticos por anamnesis, la flexión del hombro $(\sigma$ =26) presentó la mayor dispersión de datos, seguida de la inclinación lateral de la columna cervical $(\sigma=17)$ y la flexión de la columna lumbar $(\sigma=4,9)$; en cuanto a los trabajadores sintomáticos por anamnesis, presentaron una mayor dispersión en la flexión del hombro $(\sigma=4,3)$; seguida de la abducción del hombro $(\sigma=3,4)$, flexión del codo y la rodilla ( $\sigma=2,7$ cada una).

$\mathrm{Al}$ aplicar la evaluación goniométrica en el total de trabajadores a nivel de la columna cervical (flexión), se observó diferencia significativa entre los resultados obtenidos en la goniometría y lo referido durante la anamnesis $(\mathrm{p}<0,05)$. Igual comportamiento reflejó la rotación derecha e izquierda en dicho segmento corporal $(\mathrm{p}<0,05)$.

En cuanto a la columna lumbar al aplicar la técnica de goniometría se aprecia diferencia significativa con lo reportado en la anamnesis del grupo total de trabajadores en todos los movimientos que realiza dicho segmento, durante la Flexión $(\mathrm{p}<0,05)$, la Extensión $(\mathrm{p}<0,05)$, la Inclinación lateral $(\mathrm{p}<0,05)$ y, la Rotación derecha e izquierda $(\mathrm{p}<0,05$.

Asimismo, se presentó en todos los movimientos del hombro, Flexión $(\mathrm{p}<0,05$; Extensión $(\mathrm{p}<0,05)$; Rotación Interna $(\mathrm{p}<$
0,05); Rotación Externa $(\mathrm{p}<0,05)$; Abducción $(\mathrm{p}<0,05)$ y, Aducción $(\mathrm{p}<0,05)$.

Al aplicar la goniometría en el codo (Extensión), se obtiene diferencia significativa entre los resultados de la técnica goniométrica y lo expresado durante la anamnesis en el grupo total de trabajadores $(p<0,05)$.

Por otra parte, al aplicar la evaluación goniométrica en la muñeca (desviación cubital), se encontró diferencia signnificativa entre los resultados de la goniometría y lo reportado en la anamnesis del total de trabajadores $(\mathrm{p}<0,05)$.

Para finalizar, en los datos de rodilla al aplicar la goniometría (flexión), se nota diferencia significativa entre la evaluación goniométrica y la anamnesis del grupo de trabajadores examinados $(\mathrm{p}<0,05)$.

En el resto de los segmentos corporales no se obtuvieron diferencias estadísticas.

\section{DISCUSIÓN}

Los hallazgos de la presente investigación coincidieron con un estudio realizado en Taiwán en el año 2020 (14), donde el $86,42 \%$ eran hombres y $13,5 \%$ eran mujeres, con una edad promedio de 31 años.

Concordando parcialmente con un estudio realizado en una fábrica de paneles de madera (15), donde los 16 armadores evaluados con la técnica de goniometría eran hombres con un promedio de edad de 25 años y cuya sintomatología musculoesquelética fue a nivel de muñeca, región lumbar y hombros, mientras que en la presente investigación predominó el dolor en columna lumbar, cervical y muñeca respectivamente. Contrastando además con lo reportado por Chen y col. (13), donde prevaleció la sintomatología en muñecas, hombros, columna lumbar y cervical.

Los trabajadores que negaron sintomatología musculoesquelética durante la anamnesis, al realizarles la evaluación goniométrica, no presentaron limitaciones en los arcos de movilidad, resultando congruente con la ausencia de síntomas descritos en su 


\section{TABLA IV}

MEDIDAS DEL ARCO DE MOVILIDAD EN TRABAJADORES ASINTOMÁTICOS Y SINTOMÁTICOS SEGÚN ANAMNESIS.

\begin{tabular}{|c|c|c|c|}
\hline $\begin{array}{l}\text { Segmentos } \\
\text { Corporales }\end{array}$ & $\begin{array}{l}\text { Trabajadores } \\
\text { Asintomáticos }\end{array}$ & $\begin{array}{l}\text { Trabajadores } \\
\text { Sintomáticos }\end{array}$ & $\mathrm{p}^{\mathrm{a}}$ \\
\hline & $\mathrm{X} \pm \mathrm{DE}$ & $\mathrm{X} \pm \mathrm{DE}$ & \\
\hline $\begin{array}{l}\text { Columna Cervical } \\
\text { Flexión } \\
\text { Extensión } \\
\text { Inclinación Lateral } \\
\text { Rotación Derecha e Izquierda }\end{array}$ & $\begin{array}{l}33,1 \pm 1.9 \\
42,2 \pm 2,0 \\
43,3 \pm 17 \\
55,9 \pm 3,6\end{array}$ & $\begin{array}{l}28,4 \pm 0.6 \\
28,4 \pm 0,6 \\
36,6 \pm 0,8 \\
48,8 \pm 1,0\end{array}$ & $\begin{array}{l}0,000 \\
\mathrm{NS} \\
\mathrm{NS} \\
0,000\end{array}$ \\
\hline $\begin{array}{l}\text { Columna Lumbar } \\
\text { Flexión } \\
\text { Extensión } \\
\text { Inclinación Lateral } \\
\text { Rotación Derecha e Izquierda }\end{array}$ & $\begin{array}{l}77,8 \pm 4,9 \\
29,2 \pm 1,4 \\
35,1 \pm 4,0 \\
28,9 \pm 1,6\end{array}$ & $\begin{array}{l}65,0 \pm 1,4 \\
24,4 \pm 0,5 \\
32,5 \pm 0,7 \\
24,4 \pm 0,5\end{array}$ & $\begin{array}{l}0,028 \\
0,024 \\
0,000 \\
0,006\end{array}$ \\
\hline $\begin{array}{l}\text { Hombro } \\
\text { Flexión } \\
\text { Extensión } \\
\text { Rotación Interna } \\
\text { Rotación externa } \\
\text { Abducción } \\
\text { Aducción }\end{array}$ & $\begin{array}{c}148,6 \pm 26 \\
39,0 \pm 1,6 \\
62,5 \pm 3,5 \\
69,2 \pm 2,1 \\
158,1 \pm 3,1 \\
29,1 \pm 1,4\end{array}$ & $\begin{array}{l}105,7 \pm 4,3 \\
24,4 \pm 0,7 \\
48,8 \pm 2,0 \\
48,8 \pm 2,0 \\
55,8 \pm 3,4 \\
24,4 \pm 0,5\end{array}$ & $\begin{array}{l}0,000 \\
0,009 \\
0,002 \\
0,000 \\
0,012 \\
0,024\end{array}$ \\
\hline $\begin{array}{l}\text { Codo } \\
\text { Flexión } \\
\text { Extensión }\end{array}$ & $\begin{array}{c}148,6 \pm 2,7 \\
9,62 \pm 1,0\end{array}$ & $\begin{array}{c}122,0 \pm 2,7 \\
8,1 \pm 0,1\end{array}$ & $\begin{array}{c}\mathrm{NS} \\
0,000\end{array}$ \\
\hline $\begin{array}{l}\text { Muñeca } \\
\text { Desviación Radial } \\
\text { Desviación Cubital }\end{array}$ & $\begin{array}{l}28,9 \pm 1,6 \\
25,6 \pm 4,0\end{array}$ & $\begin{array}{l}24,4 \pm 0,5 \\
24,4 \pm 0,5\end{array}$ & $\begin{array}{c}\mathrm{NS} \\
0,000\end{array}$ \\
\hline $\begin{array}{l}\text { Rodilla } \\
\text { Flexión } \\
\text { Extensión }\end{array}$ & $\begin{array}{c}148,1 \pm 3,1 \\
9,8 \pm 0,6\end{array}$ & $\begin{array}{c}122,0 \pm 2,7 \\
8,1 \pm 0,7\end{array}$ & $\begin{array}{c}0,012 \\
\mathrm{NS}\end{array}$ \\
\hline
\end{tabular}

${ }^{a}$ calculada mediante t de Student. $\mathrm{NS}=$ no significativo.

anamnesis. Sin embargo, de los trabajadores que refirieron sintomatología musculoesquelética, solo un bajo porcentaje presentó limitaciones en los arcos de movilidad. Difiriendo con lo reportado por Huapaya y Gomero (16) en un personal sanitario en Lima (Perú), donde se observó que del 49\% del total de trabajadores sintomáticos por anamnesis, el $42 \%$ presentó limitaciones en los arcos de movilidad; y del 51\% que refirieron encontrarse asintomáticos, 26\% mostraron limitaciones durante la evaluación goniométrica.

Aun cuando los criterios de normalidad goniométrica no están validados antropométricamente en el Ecuador, de acuerdo a investigaciones realizadas en otros países, esta técnica de evaluación es considerada fiable y válida (17).

En relación al análisis estadístico, se obtuvo una diferencia significativa entre la evaluación goniométrica y lo reportado en

Vol. 62(1): 52 - 62, 2021 
la anamnesis en el grupo de sintomáticos y asintomáticos en un alto porcentaje de los seǵmentos estudiados, excepto en el movimiento de extensión e inclinación lateral de la columna cervical, en la flexión del codo, en la desviación radial de la muñeca y en la extensión de la rodilla. Cabe mencionar, que los resultados obtenidos por Watkins y col. (18) sugieren realizar mediciones de rango de movimientos repetidos en la rodilla para minimizar el error asociado con estas mediciones.

Ahora bien, para que la goniometría aporte información relevante, las mediciones, deben representar el valor real de la articulación medida y deben ofrecer en los ángulos articulares los mismos resultados en mediciones sucesivas en el trabajador. En este sentido, un estudio realizado por Torrealba (12), mencionó que, el marǵen de error en la precisión de las mediciones goniométricas, puede deberse a los desplazamientos segmentarios que se realizan durante la medición, a la interpretación del examinador al medir la angulación del movimiento evaluado o a la falta de dominio del examinador al momento de realizar la técnica. Norkin y White (11) han mencionado que, para alcanzar las competencias en la realización de la evaluación goniométrica, el examinador debe aprender la estructura y función de las articulaciones a evaluar y desarrollar destrezas psicomotrices para medir el grado de movilidad y la longitud muscular. Por su parte, Otoya (19) ha referido que existen factores extrínsecos (fuerza del músculo, la gravedad) e intrínsecos (propios de las articulaciones) que deben considerarse, ya que pudieran contribuir con la imprecisión durante la medición; además, Baranda (20) considera que deben incluirse otras variables (fuerza, flexibilidad) que requieren técnicas de medición no goniométricas.

Son limitantes importantes a considerar, la transversalidad del estudio, la escasa población estudiada y el realizar la evaluación goniométrica en el consultorio y no en el puesto de trabajo donde, además deben considerarse los medios de trabajo. Asimismo, debe considerarse la posibilidad de incorporar otras variables como, el tono muscular, el trofismo, la flexibilidad, la fuerza y velocidad del movimiento.

La técnica goniométrica es un método no invasivo y económico que puede realizarse en un consultorio o en el puesto de trabajo. Apoyando lo descrito por Diniz y col. (21) quienes afirman que el uso del goniómetro es un procedimiento de medición confiable y práctico que se puede aplicar fácilmente en el contexto clínico.

Dentro del examen médico ocupacional esta técnica se aplica en el examen médico pre-empleo, periódico, de egreso y para el seguimiento en casos de TME de origen ocupacional. Al no disponer de reportes investigativos nacionales en relación al tema, se podría sostener que el Ecuador carece de este tipo de investigación; además, este tipo de evaluación no se incluye dentro del protocolo de evaluación médico ocupacional ni por mandato legal ni por tradición médica. Sin embargo, en algunos países latinoamericanos como Venezuela, la aplicación de la goniometría es un requisito técnico normativo dentro de los servicios de seguridad y salud ocupacional (12).

Este estudio podría ser un primer paso de muchas otras investigaciones que permitan considerar el incorporar la técnica goniométrica como estrategia para el enfoque preventivo en la vigilancia de la salud de los trabajadores, reduciendo costos por estudios de imágenes, ausentismo e indemnización por TME, tanto a las empresas privadas como a los sistemas de salud gubernamental.

\section{AGRADECIMIENTO}

Cabe mencionar que el presente estudio sirvió como trabajo de grado del principal autor, Jean Pino-Sánchez, para su titulación como Maǵister en Salud y Seguridad Ocupacional. 


\section{REFERENCIAS}

1. Gutiérrez A. Guía téenica para análisis de exposición a factores de riesgo ocupacional para el proceso de evaluación en la calificación de origen de la enfermedad profesional [Internet] Colombia: Ministerio de la Protección Social; 2011 [cited 2020 Oet 29]. Disponible en: https://comunicandosalud. com/wpcontent/uploads/2019/06/guia_exposicion_factores_riesgo_ocupacional.pdf.

2. Comisión Obrera de Castilla y León. Manual de trastornos musculoesqueléticos. 2th.ed. España: Secretaria de Salud Laboral CG.OO; 2010.

3. European Agency for Safety and Health at Work. European Risk Observatory Report. OSH in figures: Work-related musculoskeletal disorders in the EU-Facts and figures [Internet] Luxembourg: Publications Office of the European Union; 2010 [cited 2020 Oct 27]. Available from: https://osha. europa.eu/en/publications/osh-figureswork-related-musculoskeletal-disorders-eufacts-and-figures.

4. Bellorín M, Sirit Y, Rincón C, Amortegui M. Síntomas músculo esqueléticos en Trabajadores de una empresa de construcción civil. Salud Trab 2007; 15 (2): 89-98 [cited 2020 Nov 24]. Disponible en: redalyc.org/ pdf/3758/375839287003.pdf.

5. Agencia Europea para la Seguridad y la Salud en el Trabajo. Magazine [Internet] 2000 [Consultado 10 Oct 2030]; 3. Disponible en: http://www.exyge.eu/blog/ wp-content/uploads/2013/11/Magazine_3_Prevencion_de_los_trastornos_musculoesqueleticos_de_origen_laboral.pdf.

6. Reglamento de Seguridad y Salud de los Trabajadores y Mejoramiento del Ambiente de Trabajo. Decreto Ejecutivo 2393 [Internet] Ecuador: Registro Oficial Suplemento 565; 1986. [cited 2020 Oct 25]. Disponible en: http://www.trabajo.gob.ee/wpcontent/ uploads/downloads/2012/12/Reǵlamentode-Seguridad-y-Salud-de-los Trabajadores-yMejoramiento-del-Medio-Ambiente-de-Trabajo-Decreto-Ejecutivo-2393.pdf.

7. Reglamento para el Funcionamiento de los Servicios Médicos de Empresas. Acuerdo Ministerial 1404 [Internet] Ecuador: Registro Oficial Suplemento 698; 1979. [cited
2020 Oct 15]. Disponible en: http://www. trabajo.gob.ec/wp-content/uploads/downloads/2012/12/Reglamento-para-el-Funcionamiento-de-Servicios-M\%C3\%A9dicosAcuerdo-Ministerial-1404.pdf.

8. Instituto Ecuatoriano de Seguridad Social. Estadístico B. Ecuador: Dirección Actuarial y de Investigación; 2013.

9. Oliveira I, Rodríguez G. El dolor de espalda en funcionarios que trabajan en oficinas de la administración pública en la Provincia de Pontevedra. TRANCES: Revista de Transmisión del Conocimiento Educativo y de la Salud 20146 (2); 75-90. [Consultado 15 de sept 2020] .Disponible en: https://dialnet.unirioja.es/servlet/ articulo? codigo $=6425903$

10. Gómez-García A, Merino-Salazar P, Espinoza-Samaniego C, Cajías Vasco P. I Encuesta sobre seguridad y salud en el trabajo en Quito: siniestralidad laboral. Pódium 2018 ; 33: 25-54. [consultado 17 Sept 2020] Disponible en: https://doi. org/10.31095/podium.2018.33.3

11. Joyce W, Cynthia N. Capítulo 1. Conceptos básicos. Capítulo 2. Procedimientos. En Davis Company, F. A., Eds. Medición del movimiento articular: una guía para la goniometría; 1995. p. 3-19-24.

12. Taboadela CH. Goniometría: Una herramienta para la evaluación de las incapacidades laborales. Buenos Aires: Asociart ART; 2007.

13. Torrealba F. Aplicaciones de la goniometría en la gestión de la salud ocupacional en Venezuela. Salud Trab 2017; 25 (2): 167-174 [cited 2020 Oet 18]. Disponible en: http:// servicio.bc.uc.edu.ve/multidisciplinarias/ saldetrab/Vol25n2/art06.pdf.

14. Yi-Lang Ch, Yan-Ting $Z$, Bang-Nan L, ChihChuan Y. Musculoskeletal disorders symptoms among Taiwanese bakery workers. Int J Environ Res. Public Health 2020; 17 (8): 2960.

15. Clark N. Estudio ergonómico del proceso de armado y encolado de paneles. Rev Ergon Invest Desar 2019; Vol 1 (3): 138-157.

16. Huapaya C, Gomero R. Evaluación postural y presencia de dolor osteomuscular en trabajadores de una clínica materno-infantil, en la ciudad de Lima Perú. Rev Med Hered $2018 ; 29: 17-21$. 
17. Gogia P, Braatz J, Rose S, Norton B. Reliability and validity of goniometric measurements at the knee. Physical Therapy 1987; 67 (2): 192-195. Available from: https:// doi.org/10.1093/ptj/67.2.192.

18. Watkins MA, Riddle DL, Rothstein JM, Lamb RL. Goniometric reliability in a clinical setting: Shoulder measurements. Physical Therapy 1987; 67(5): 668-673.

19. Otoya W. Manual de Kinesiología Aplicada. Lima: Organización Panamericana de la Salud; 2000 [cited 2020 Oet 29]. http://www. bvsde.paho.org/texeom/manualesMEC/kinesiologia.pdf? $\mathrm{ua}=1$.
20. Baranda-Andujar P. Movilidad articular y estiramientos en Salas de Musculación . 2015 [cited 2020 Oet 10]. https://www. researchgate.net/publication/266455024.

21. Diniz KMA, Freire R, Bittencourt NFN, Mendonça LDM. Correlation between goniometric and photogrammetric assessment of shank-forefoot alignment in athletes. Foot 2020; 45. 101687 [consultado 17 Sept 2020], doi: 10.1016/j. foot.2020.101687. 